\title{
Microcalcification Detection by Morphology, Singularities of Contourlet Transform and Neural Network
}

\author{
Rekha Lakshmanan and Vinu Thomas
}

\begin{abstract}
The proposed method presents a new classification approach to microcalcification detection in mammograms using morphology, Contourlet Transform and Artificial Neural Network. Early detection of breast cancer is possible by enhancing microcalcification features obtained using morphology and singularities of Contourlet Transform. The significant edge information indicating the relevant features in various decomposition levels are preserved while removing the artifacts. These features are utilized to detect microcalcifications by classification employing the Back Propagation Neural Network. Target to background contrast ratio, Contrast and Peak Signal to Noise ratio are considered for performance evaluation of the enhancement algorithm. The accuracy of the classification algorithm is $95 \%$. The miniMIAS mammographic database is employed for testing the accuracy of the proposed method and the results are promising.
\end{abstract}

Keywords--- Breast Cancer, Back Propagation Neural Network, Contourlet Transform, Morphology

\section{INTRODUCTION}

$\mathrm{B}$ REAST cancer is one of the leading causes of cancer death among women. According to the statistics in 201112 , the expected mortality rate due to breast cancer is about 39,520 [1], even though there is decrease in death rates since 1990. According to the Indian Council of Medical Research (ICMR), breast cancer becomes the leading cause of cancerrelated mortality among women and it will affect nearly 0.25 million women in India by 2015. Mammography as a screening tool is one of the best proven technique for early breast cancer detection. Mammographic image analysis is a complicated and difficult task which requires opinion of highly trained radiologists. Detection of MiCro-Calcification (MCC), a possible symptom of breast cancer is a complex task because of the inhomogeneous background and the high noise level in the images due to emulsion artifacts. Microcalcifications are tiny, granular, linear, or irregular deposits of Calcium Phosphate Hydroxide which appear as bright white spots with size ranging from $0.1-1.0 \mathrm{~mm}$ and an average diameter about 0.3 [2].A high contrast is essential in

Rekha Lakshmanan, Assistant Professor at K.M.E.A Engineering College, Aluva, Ernakulam

Vinu Thomas, Associate Professor in Govt. College of Engineering, Cherthala

DOI: $10.9756 /$ BIJNTA.3086 differentiating minute MCC structures with breast tissues. Computer aided image processing techniques assists radiologists in analyzing suspicious areas and to provide a second opinion of diagnosis. The multi scale and multidirectional analysis of the proposed method can effectively enhance sharp variation points indicating the presence of MCC by removing various artifacts thereby increasing the reduction of false positives.

\section{LITERATURE SURVEY}

Recently, wavelet-based enhancement approaches [3][4] [5][6] have been employed to acquire better performances. Natural images have higher geometrical characteristics [7], and the discontinuity is always along the smooth curve. The sharp edges and singularities in natural images cannot be efficiently presented by a separable two dimensional wavelet transform even though it provides an optimal representation for one dimensional piece-wise smooth signals. To overcome the drawbacks of Wavelet Transform, Contourlet transform by Minh Do et al [8], an efficient representation is employed to capture the smooth contours that are the dominant feature in natural images. The multi resolution property of Contourlet transform is proficient in separating small objects such as microcalcifications from large objects such as background structures. Manzano et.al [9], proposed an image enhancement technique using an orientation space analysis based on a contourlet transform. Xinsheng Zhang and Hua Xie [10] utilized Contourlet Transform, Generalized Gaussian Mixture Model (GGMM) and Bayesian classifier to enhance the suspicious features. $\mathrm{Hu}$ et.al. suggested a detection Algorithm[11] of Suspicious Lesions by Adaptive Thresholding Based on Multi resolution Analysis along with morphological filter to remove the noise and to enhance the gray-level feature and shape feature.Wiselin Jiji.G [12] utilized features from wavelet decomposition and Gabor filter for detecting micro calcification using back propagation neural network. Manimegalai.P[13] developed a system extracting statistical features by wavelet decomposition for classifying breast tissue using Back propagation Neural Network (BPNN). Leena Jasmine [14] used a new approach for micro calcification detection using back propagation neural network and non subsampled Contourlet transform which yielded a significant true detection rate approximately $87 \%$.

\section{METHODOLOGY}

The proposed algorithm makes use of the multiscale and directionality properties of the Contourlet Transform to 
enhance clinically important microcalcification features in mammograms while suppressing artifacts such as those due to emulsion discontinuity. These features are utilized for microcalcification detection using Back Propagation neural network [14]. Here we employ Top-Hat operation in morphology as well as selective pruning of the modulus maxima coefficients at different levels of resolutions of the contourlet decomposition of a mammogram to enhance microcalcification features. For selectively pruning the modulus maxima coefficients, the parent-child relationship among the directional filter bank outputs in the filter bank implementation at different levels of the contourlet decomposition is employed. The significant coefficients that correspond to significant features of the image are retained, while leaving out those that possibly correspond to image noise, such as those due to emulsion discontinuity. The purpose of the proposed method is to enhance suspected microcalcifications so that early stage diagnosis and treatment can be done in effective manner.

\subsection{Contourlet Transform}

The Contourlet transform, an efficient two dimensional transform using multiscale and directional filter banks can be considered as an extension of Wavelet transform. The directional information in the regular 2-D wavelet transform is confined only to the vertical, horizontal and diagonal directions [15].The nonseparable basis functions of Contourlet transform are oriented at various directions in multiple scales, with flexible aspect ratios. They offer a high degree of directionality. Two dimensional wavelets are effective in isolating edge discontinuities, but an efficient detection of smoothness along the contours is not possible. It is possible to capture the geometrical smoothness of the contour along any possible direction. The implementation of Contourlet Transform consists of two stages: multiscale decomposition and multidirectional decomposition. Laplacian Pyramid (LP) proposed by Burt and Adelson [16] is used for multiscale decomposition where a sampled low-pass result of original image and the difference between the original and the prediction are generated as shown in figure 1 .

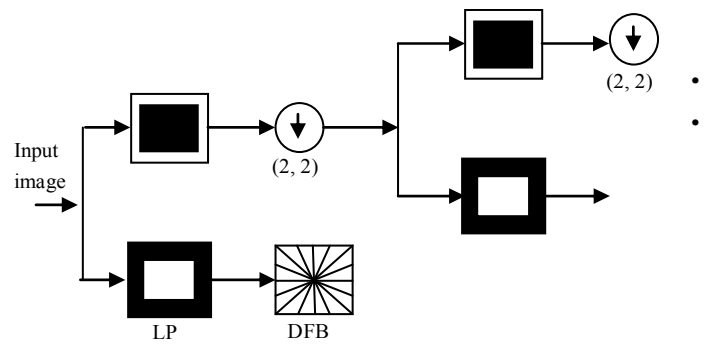

Figure 1: Block Diagram of Contourlet Transform

A coarse approximation is obtained by decimating a low pass filtered version of original image. A prediction image is generated by interpolating a low pass filtered version of the coarse approximation. A band pass image is obtained by subtracting prediction image from original image. The coarse image is utilized for the iterative process of the contourlet transform to obtain coarser approximations and directional information at coarser resolutions. The band pass image is given as input to the Directional Filter Bank (DFB) proposed by Bamberger and Smith[17]which is implemented by an mlevel binary tree decomposition using quincunx filter banks with diamond-shaped fan filters leading to $2^{\mathrm{m}}$ subbands with wedge-shaped frequency partitioning. The DFB is constructed from two building blocks. The first building block, a twochannel quincunx filter bank with fan filters divides the 2-D spectrum into two directions, horizontal and vertical. Reordering of image pixels using a shearing operator is performed as the second block. The generation of four subbands after the first two levels is shown in figure 2. A subband at the second level is generated using multirate identity. The fan filters at the second level with the sampling matrix Q0 at the first level generates an equivalent filter with a quadrant frequency response. From third level subbands onwards, a resampling operation on quincunx filterbank is performed utilizing multirate identity to obtain parallelogram filters.

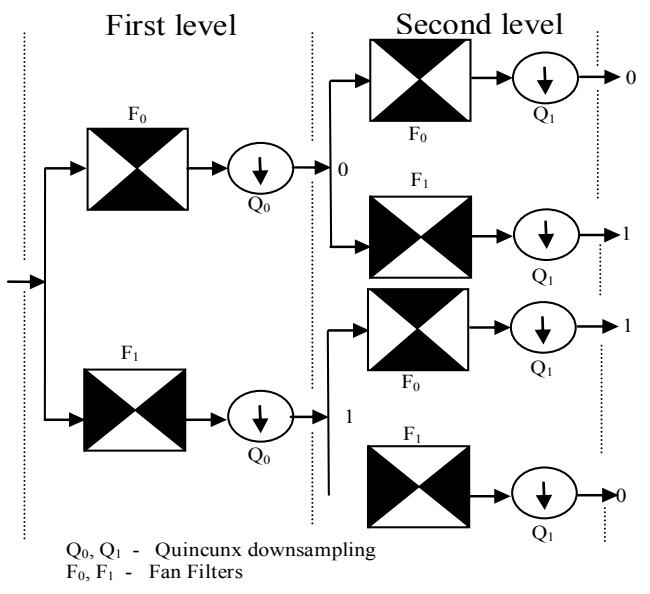

Figure 2: The Directional Filter Bank Structure

Efficiency of the proposed methods for enhancement and denoising are based on the preservation of directional information in Contourlet transform. Impulse response of a bandpass arm at one level of contourlet decomposition constitutes the combined impulse responses of filters in LP as well as DFB. The impulse responses of all the bandpass arms can be obtained by considering an input image with a single white pixel centered in a black background as shown in figure 3.

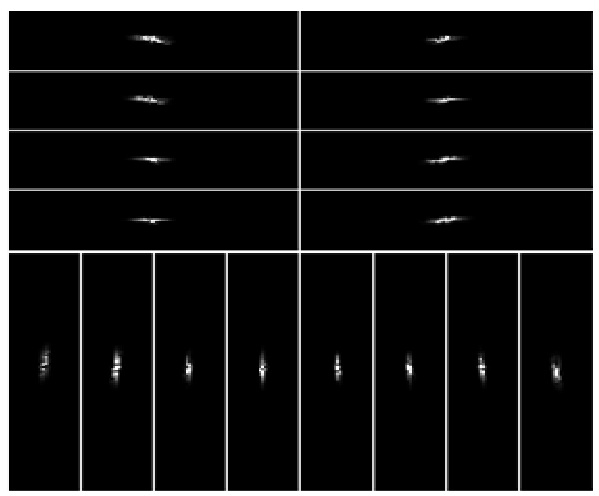




$$
\begin{aligned}
& I \circ S=(I \Theta S) \oplus S \\
& I \bullet S=(I \oplus S) \Theta S
\end{aligned}
$$

Opening operation helps to keep the background (i.e., features that cannot hold the structuring element) which is not required for the proposed method. So a Top-Hat operation $[20]$ is performed to remove the background in order to get the required microcalcification features. Top-Hat representation is given by

$$
T H=I-(I \circ S)
$$

In Top-Hat operation, the foreground objects can be highlighted by suppressing the dark background. Since the low contrast MCCs in mammograms appear as circularly bright spots, and a calcification has approximately a size of 20 pixels on each mammogram [21], the proposed method considers a structuring element larger than 20 pixels. So the detection of microcalcification is possible through morphological approach. The resulting image is converted to a binary image by thresholding it with the value of $8^{*} \sigma$ (empirically obtained), where $\sigma$ is the standard deviation of the result image.

\subsection{Proposed Method}

The proposed method combines the approach based on the modulus maxima of the contourlet transform and the morphological approach by a logical AND operation to detect clinically important microcalcification structures while removing noise due to emulsion artifacts. The low pass filters employed at the LP stage are derived from the PKVA filters [22] and the fan filters at the DFB stage are derived from the BIOR 9, 7 filters [23]. The pixels in the mammogram image corresponding to the location of logic 1 in the image obtained from the previous step are boosted to emphasize the locations of the microcalcifications. To develop a CAD technique that classifies the mammographic images into normal and those with microcalcifications, a Back Propagation Neural Network (BPNN) [24] is employed. For the training set, the ground truth that accompanies the MIAS database [25] is employed. 256 x 256 snippets of the mammogram images are employed for training the Back Propagation Neural Network. Five hundred such blocks were employed in the training set. In order to reduce the dimension of the feature vector, the enhanced image is divided into blocks of size $32 \times 32$. The energy of each block of the denoised and quality enhanced image is computed by squaring every element in the block and adding. In order to simplify the computation, energy value of each block is divided by the maximum energy value of all blocks to get normalized energy values which are less than or equal to one. The lexicographically ordered 64 x 1 energy vector is computed for each mammogram snippet and is considered as the feature vector for the classification process. The BPNN used for the classification thus employs 64 input neurons and one output neuron. Five hidden layer neurons in a single hidden layer were employed for the BPNN. For the test data set 256 x 256 snippets of mammograms similarly enhanced as before, are converted to equivalent $64 \times 1$ feature vectors. 


\subsection{Algorithm}

1. Morphological Operation: Top-Hat operation of the original mammogram image is performed (a $256 \mathrm{x}$ 256 snippet is taken here) to remove the background in order to get the required microcalcification features.

2. Thresholding: The resultant image is thresholded using $8^{*} \sigma$, where $\sigma$ is the standard deviation of the resultant image.

3. Coefficient Extraction: Contourlet Transform of original image is computed for three scales with a four level directional decomposition at each scale.

4. Singularity detection: Modulus -Maxima of Contourlet coefficients at directional subbands at each level of resolution in the specified directions are computed.

5. Artifact reduction: To remove artifacts, coefficients that hold parent-child relationships from finer to coarser levels are retained and others are pruned out.

6. Boosting: The modulus maxima that are retained after step 5 and the coefficients on their either sides are boosted.

7. Coarse suppression: Assign zero arrays to the coarse subband. Apply Pyramidal directional filter bank reconstruction on the selected coefficients in the directional subbands.

8. Thresholding: Threshold the resultant image using the value $8^{*} \sigma$, where $\sigma$ is the standard deviation.

9. Logical AND ing: Apply a logical AND operation with the results obtained from step 2 and step 8 to locate the MCCs.

10. Image enhancement: The pixels in the mammogram image corresponding to the location of logic 1 in the image obtained from the previous step are boosted to emphasize the locations of the microcalcifications.

11. Feature Reduction: Divide the enhanced image into blocks of size $32 \times 32$ to reduce the size of the feature vector.

12. Feature Vector generation: Calculate the energy of each block and normalize it to generate the $64 \times 1$ feature vector.

13. Classification: Train the BPNN using the training set. Each test datum element ( $256 \times 256$ mammogram snippet) is similarly converted to a $64 \mathrm{x} 1$ energy vector as before, prior to presentation to the BPNN for classification.

\section{Database Used}

The proposed method was tested on mini-MIAS database [25] of digital mammograms by UK research group. The database consists of 322 images of size 1024 x 1024 and 256 gray levels. Among them, 25 images contain microcalcifications. Among these 25 images, 13 cases are diagnosed as malignant and 12 as benign.

\section{Performance Measures}

Various performance measures such as Contrast, Peak Signal to Noise Ratio and Target to Background Contrast ratio[26] were considered for measuring the efficiency of the proposed method.
The contrast, $\mathrm{C}$ of a region is defined by

$$
C=\frac{(f-b)}{(f+b)}
$$

where $f$ is the mean gray-level value of the foreground and $b$ is the mean gray-level value of the background. PSNR is defined as

$$
P S N R=\frac{(p-b)}{\sigma}
$$

where $\mathrm{p}$ is the maximum gray-level value and $\sigma$ is the standard deviation of the background.

In order to evaluate the effect of visual appearance of our method, we consider the Target to Background Contrast ratio using Variance $\left(\mathrm{TB}_{\mathrm{C}}\right)$. The expression for computing $\mathrm{TB}_{\mathrm{C}}$ is

$$
T B_{c}=\frac{\delta_{\mu}}{\sigma}
$$

where $\delta_{\mu}$ is the difference between the ratios of the mean gray levels in the foreground and background and is the ratio of standard deviation of enhanced and original image.

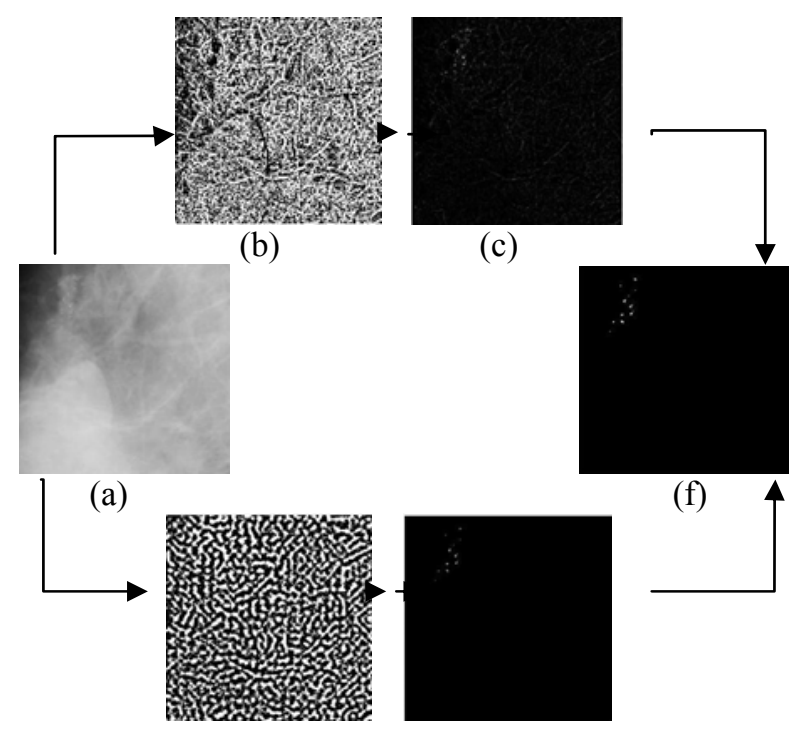

Figure 4: (a) Original Image, (b) Tophat Transformed Image (c) Image after Thresholding Tophat Transformation,(d) Coarse Suppressed contourlet Reconstructed Image, (e) Thresholded Image after Coarse Suppressed Contourlet

Reconstruction, (f) Logical AND-ing of Thresholded Images obtained after Morphological and Contourlet Transform.

\section{EXPERIMENTAL RESULTS}

The mini-MIAS mammographic database was employed for benchmarking the proposed algorithm. Three levels of the Laplacian Pyramid with four levels of the Directional Filter Bank (i.e., 16 directional subbands) at each level of the LP decomposition were employed for the contourlet decomposition of the mammogram images. The PKVA filters were employed for the Laplacian Pyramid and BIOR 9, 7 filters were employed at the DFB stage. Figure 4 shows the results of the algorithm on the image MDB 241 from the mini- 
MIAS database, for the major intermediate steps of the proposed algorithm. The enhanced results for the Mdb241, Mdb211, Mdb245 and Mdb249 images from the mini MIAS database are shown in figure 5. Mdb249 is a mammographic image having dense glandular background tissue with welldefined malignant microcalcifications. Mdb211 is a fatty glandular mammogram with a difficult to detect microcalcification cluster. Mdb245 represents a mammographic image with widely distributed calcifications. Mdb241 is a mammographic image having easy to detect microcalcifications. The results in figure 5 reveal that the proposed technique enhances the appearance of the microcalcifications against the surrounding dense tissue which may otherwise obscure these structures.
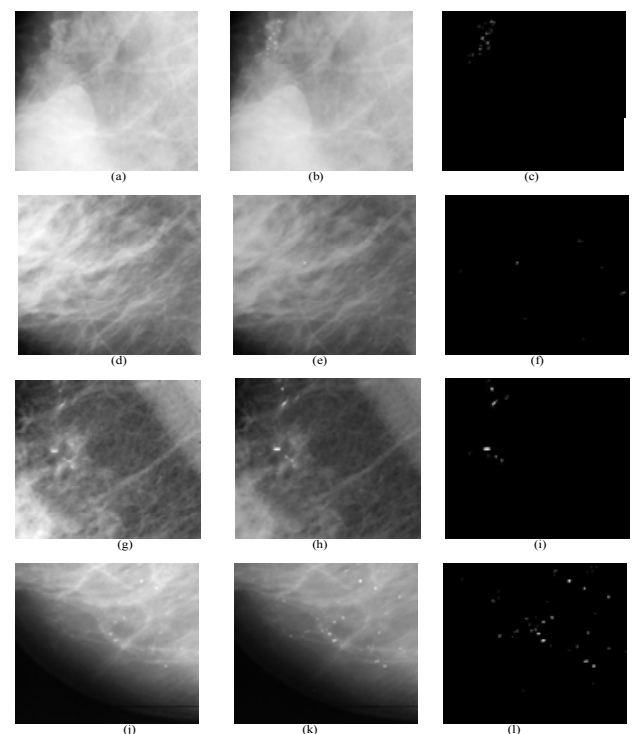

Figure 5: The Enhancement and Segmented Results for the Microcalcification Features on Selected ROI's : (a)(d)(g)(j)

Original ROI of mdb241, mdb249, mdb245, mdb211,

(b)(e)(h)(k) Enhancement by Proposed Method (c)(f)(i)(l) Segmented Microcalcifications

The quantitative results of figure 6 substantiate the improvement in contrast and visibility with reduction in noise for the proposed technique. High value of PSNR indicates the good performance of the enhancement technique. The high $\mathrm{TBc}$ value indicates the improvement in the visual appearance of the enhanced image as well as algorithm efficiency. The significant improvement in the values of TBc and Contrast with the proposed method indicates that the enhanced image is visually superior with a larger contrast. In order to train the neural network, the images are randomly selected. The proposed method included 3008 blocks of mammographic images for training the network. Validation of the proposed method was conducted by the ground truth and the advice of expert radiologists provided in the database. Proposed method yields $95 \%$ true positive rate and $96.71 \%$ true negative rate with $96.70 \%$ accuracy. Since the false positive rate is very less $(3.29 \%)$, the proposed method is very helpful reducing unnecessary biopsies.

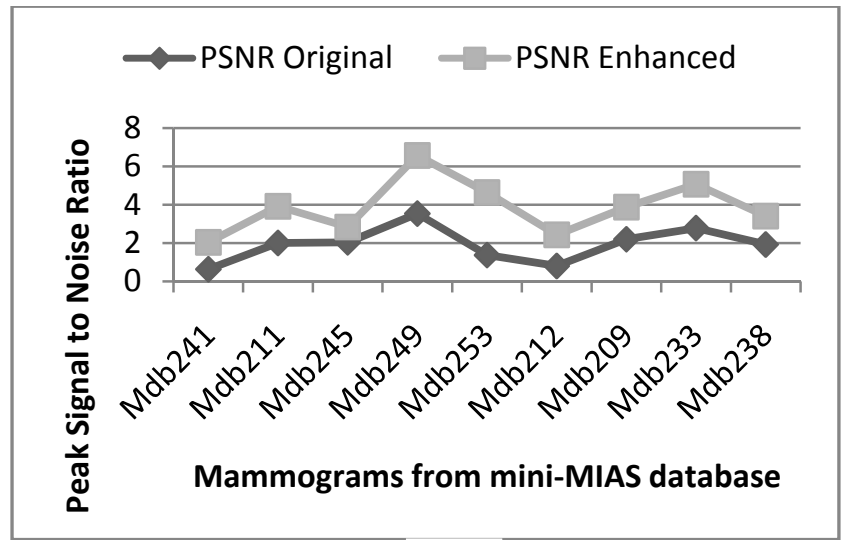

(a)

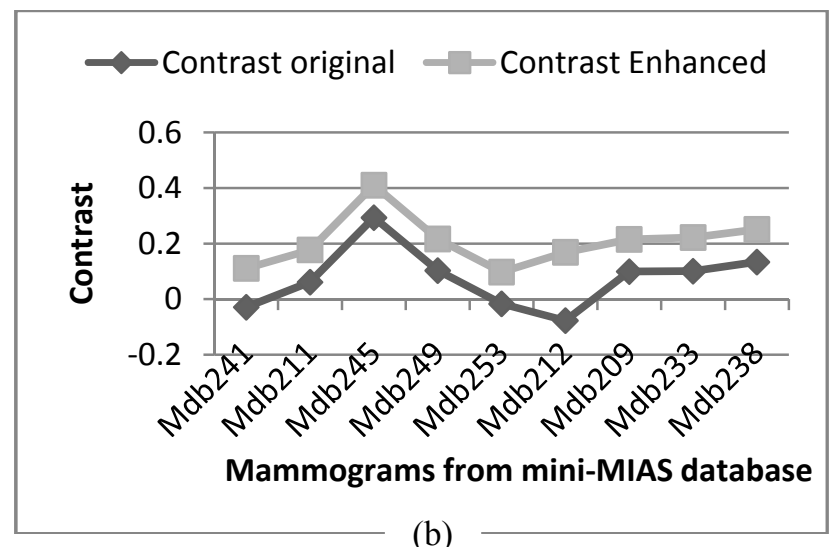

(b)

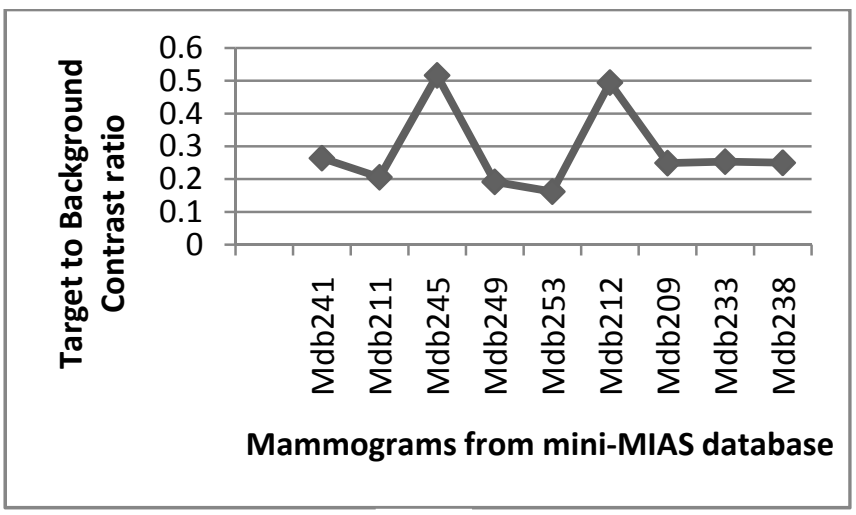

(c)

Figure 6: Comparison of Performance Measures,(a)PSNR, (b)Contrast, (c) TBc of Original and Enhanced Image

\section{CONCLUSION}

In this paper, a fast, novel method is proposed based on morphological operation and selected contourlet coefficients for mammographic image enhancement. Top-Hat morphological operation reduces the dark background while improving foreground microcalcification features. Directional subband coefficients obtained from contourlet transform on either side of the selected coefficients in the corresponding tree were boosted while those that exist at only the finest scales were suppressed. Relevant edge features including microcalcification structures were enhanced while image 
artifacts that exist at the finest scales were suppressed. This method assists radiologists in providing a second opinion for detecting breast cancer in its early stage. Quality measures including contrast, PSNR and TBc were calculated to determine the efficiency of the proposed method. Experimental results showed that the resultant enhanced images using the proposed method are effective in improving the contrast and visual appearance by reducing artifacts. Accuracy of the proposed method is found to be $96.7 \%$.

\section{REFERENCES}

[1] ACS, Cancer facts and figures, 2010.

[2] R.N. Strickland and Hee. Il. Hahn, "Wavelet transforms for detecting microcalcifications in mammograms", IEEE Transactions on Medical Imaging, Volume. 15, No.2, Pp. 218 - 229, April 1996.

[3] L. Li, W. Qian and L.P. Clark, "X-Ray medical image processing using directional wavelet transform", Proc. IEEE Int. Conf. Acoustics, Speech and Signal Processing, Volume.4, Pp. 2251-2254, 1996.

[4] G. Lemaur, K. Drouiche and J. DeConinck, "Highly regular wavelets for the detection of clustered microcalcifications in mammograms", IEEE Trans. Med. Imag., Volume. 22, No. 3, Pp. 393-401, March 2003.

[5] S.G. Mallat, "A theory for multiresolution signal decomposition: The wavelet representation", IEEE Transations on Pattern Analysis and Machine Intelligence, Volume. 11, No. 7, July 1989.

[6] M.G. Mini, V.P. Devassia and T. Thomas, "Multiplexed wavelet transform technique for detection of microcalcification in digitized mammograms", J. Digit. Imag., Volume. 17, No. 4, Pp. 285-291, 2004.

[7] D.H. Hubel and T.N. Wiesel, "Receptive fields, binocular interaction and functional architecture in the cat's visual cortex", J. Physiol., No.160, Pp. 106-154, 1962.

[8] M.N. Do, "Directional Multiresolution Image Representations", Ph.D. Thesis, EPFL, Lausanne, Switzerland, Dec. 2001.

[9] J.A. Manzano-Lizcano, C. Sánchez-Ávila and L. Moyano-Pérez,”A Microcalcification Detection System for Digital Mammography using the Contourlet Transform ",International Conference on Computational \& Experimental Engineering \& Science, Madeira, Portugal, 2004.

[10] Xinsheng. Zhang and Hua Xie, " Mammograms Enhancement and Denoising Using Generalized Gaussian Mixture Model in Nonsubsampled Contourlet Transform Domain", Journal Of Multimedia, Volume. 4, No. 6, 2009.

[11] Kai. Hu, Xieping. Gao, and Fei. Li, "Detection of Suspicious Lesions by Adaptive Thresholding Based on Multiresolution Analysis in Mammograms", IEEE Transactions on instrumentation and measurement, Volume. 60, No. 2, February 2011.

[12] J. Dheeba and G. Wiselin. Jiji, "Detection of Micro calcification Clusters in Mammograms using Neural Network", International Journal of Advanced Science and Technology, Volume. 19, Pp. 13-22, June, 2010.

[13] P. Manimegalai, P. Revathy and Dr.K. Dhanushkodi, "Microcalcification Detection In Mammogram Image Using Wavelet Transform And Neural Network", International Journal Of Advanced Scientific Research And Technology, Volume 1, No.2, February, 2012.

[14] J.S. Leena. Jasmine, A. Govardhan and S. Baskaran, "Classification of Microcalcification in Mammograms using Nonsubsampled Contourlet Transform and Neural Network", European Journal of Scientific Research, Volume.46 No.4, Pp.531-539, 2010.

[15] M.N. Do and M. Vetterli, "The contourlet transform: an efficient directional multiresolution image representation," IEEE Trans. of Image Processing, Volume.14, No.12, Pp. 2091-2106, Dec. 2004.

[16] P.J. Burt and E.H. Adelson. "The Laplacian pyramid as a compact image code '’. IEEE Trans. Commun., Pp.532-540, April 1983.

[17] R.H. Bamberger and M.J.T. Smith, "A filter bank for the directional decomposition of images: Theory and design", IEEE Trans. Signal Proc., Volume. 40, No. 4, Pp. 882-893, April 1992.

[18] D.D.Y Po and M.N. Do, "Directional multiscale modeling of images using the contourlet transform," IEEE Trans. on Image Processing, Volume. 15, No. 6, Pp. 1610 - 1620, 2006.

[19] Michael. Wirth, Matteo. Fraschini, and Jennifer. Lyon, "Contrast enhancement of microcalcifications in mammograms using morphological enhancement and non-flat structuring elements",
Proceedings of the $17^{\text {th }}$ IEEE Symposium on CBMS'04, Pp.1063-7125, 2004.

[20] F. Meyer, "Contrast feature extraction", J.L. Chermant (Ed.) Quantitative Analysis of Microstructures in Material Sciences, Biology and Medicine, Riederer Verlag, Stuttgart, Germany, 1978.

[21] Wan. Mimi. Diyana, Julie. Larcher, and Rosli. Besar,."A comparison of clustered microcalcifications automated detections in digital mammogram", Proc. Of ICASSP'03, Volume.2, Pp.385-388, 2003.

[22] S.M. Phoong, C.W. Kim, P.P. Vaidyanathan and R. Ansari., "A new class of two-channel biorthogonal filter banks and wavelet bases," IEEE Trans. Signal Proc., Volume. 43, No. 3, Pp. 649-665, 1995

[23] M. Vetterli and C. Herley, "Wavelets and filter banks: Theory and design", IEEE Trans. Signal Proc., Volume. 40, No. 9, Pp. 2207-2232, 1992.

[24] S.N. Sivanandam, Introduction to Neural Networks using MATLAB 6.0, Tata Mc-Graw Hill, 2006.

[25] J. Suckling," Mammographic Image Analysis Society Database", available online: http://peipa.essex.ac.uk/ipa/pix/mias , 1994.

[26] Rekha Lakshmanan, Rahila Ramesh and Vinu Thomas, "Enhancement of Microcalcification Features in Mammograms using Zero-Crossings of the Contourlet Transform, "Journal of Emerging Trends in Engineering and Applied Sciences (JETEAS), Volume 2, No. 6, Pp.1009-1014, 2011.

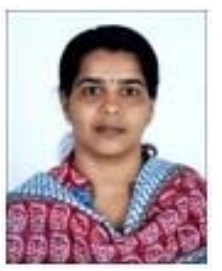

Rekha Lakshmanan is presently pursuing her $\mathrm{PhD}$ at Govt. Model Engineering College, Kochi, India. She completed her M-Tech in Digital Image Computing from the University of Kerala in 2008 and B-Tech in Computer Science and Engineering from the Cochin University of Science and Technology (CUSAT) in 2001. Her area of research interest is Image Processing. She has 4 papers in international journals 2 papers in national conferences and 4 papers in international conferences to her credit. Currently she is working as Assistant Professor at K.M.E.A Engineering College, Aluva, Ernakulam.

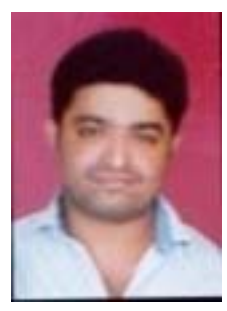

Vinu Thomas is at present, working as Associate Professor in Govt. College of Engineering, Cherthala, managed by the Institute of Human Resource Development, Govt. of Kerala, India. He was awarded $\mathrm{PhD}$ in Electronics (Development of algorithms for inverse scattering in Microwave Imaging) by the Cochin University of Science and Technology (CUSAT) in 2009. He secured the Gold Medal and first rank for MTech in Electronics from the Department of Electronics, CUSAT, for 1999-2001. He did his B-Tech degree in Electronics and Communication Engineering from Mar Athanasius College of Engineering, Kothamangalam, Kerala, during 1989-1993. He has 18 papers in international journals, 11 papers in international conferences and 5 papers in national conferences to his credit. 